\title{
Linear Programming Models for Jamming Attacks on Network Traffic Flows
}

\author{
Patrick Tague, David Slater, and Radha Poovendran \\ Network Security Lab (NSL), Department of Electrical Engineering \\ University of Washington, Seattle, WA, USA. \\ Email: \{tague, dmslater, rp3\}@u.washington.edu
}

\author{
Guevara Noubir \\ College of Computer and Information Science \\ Northeastern University, Boston, MA, USA. \\ Email: noubir@ccs.neu.edu
}

\begin{abstract}
We present a new class of network attacks, referred to as flow-jamming attacks, in which an adversary with multiple jammers throughout the network jams packets to reduce traffic flow. We propose a linear programming framework for flowjamming attacks, providing a foundation for the design of future protocols to mitigate flow-jamming. We propose metrics to evaluate the effect of a flow-jamming attack on network flow and the resource expenditure of the jamming adversary. We develop, evaluate, and compare a variety of flow-jamming attacks using the proposed metrics and the linear programming formulation. In addition, we formulate two approaches for distributed flow-jamming attacks for a set of jammers operating without centralized control and compare the performance to the centralized attacks using the linear programming formulation.
\end{abstract}

\section{INTRODUCTION}

The nature of wireless communication using an open and shared physical medium makes it vulnerable to denial-ofservice (DoS) attacks [1]. A jamming adversary can perform a variety of DoS attacks, such as transmitting wide-band noise, high-power narrow-band pulses, or interfering waveforms [2]. Anti-jamming communication systems typically rely on the use of spread-spectrum techniques, forcing the adversary to jam a wider frequency band and significantly increasing the jamming power [2], [3]. Such techniques are especially effective against resource-constrained jamming adversaries, as the required energy to jam each bit is drastically increased.

A resource-constrained jamming adversary can, however, counteract the impact of an anti-jamming system such as spread-spectrum by incorporating information of higher-layer communication or networking protocols. For example, intelligent jamming techniques have recently been developed for DoS attacks targeting certain wireless link layer and MAC

Work of G. Noubir was performed while visiting the Network Security Lab at the University of Washington.

This work was supported in part by the following grants: ONR YIP, N00014-04-1-0479; ARO PECASE, W911NF-05-1-0491; ARL CTA, DAAD19-01-2-0011; and ARO MURI, W911NF-07-1-0287.

This document was prepared through collaborative participation in the Communications and Networks Consortium sponsored by the US Army Research Laboratory under the Collaborative Technology Alliance Program, DAAD19-01-2-0011. The US Government is authorized to reproduce and distribute reprints for Government purposes notwithstanding any copyright notation thereon. The views and conclusions contained in this document are those of the author and should not be interpreted as representing the official policies, either expressed or implied, of the Army Research Laboratory or the US Government. protocols [4]-[6] and link layer error correction protocols [7], leading to significant energy savings over continuous jamming.

We suggest that DoS attack efficiency can be further improved by incorporating network layer information. Since a single packet traverses multiple wireless network links, the adversary can choose to jam each packet when minimal energy is required, effectively jamming the traffic flow [8]. An adversary in control of multiple jammers can thus balance the total energy expenditure required to jam network flows over the jammers, optimizing an objective function such as the total energy and prolonging jammer lifetime. Hence, the efficiency of the attack can be optimized by intelligent assignment of jammers to flows. We refer to this efficient DoS attack as a flow-jamming attack.

The first step toward defending against flow-jamming attacks is the ability to model them in the context of network protocol design. To the best of our knowledge, incorporating the effects of jamming into network protocol design is a new research area. We make the following contributions toward this problem.

- We show that flow-jamming attacks can be formulated using a linear programming framework, often used for network resource allocation problems.

- As the basis of our formulation, we propose metrics to evaluate the effect of flow-jamming attacks on network traffic flows and the resource expenditure of the jamming adversary with respect to a finite resource constraint.

- We develop, evaluate, and compare a variety of flowjamming attacks which are optimal with respect to the proposed metrics.

- We propose two approaches for distributed flow-jamming attacks for a set of jammers without centralized control and compare the performance of the distributed and centralized attacks.

The remainder of this work is outlined as follows. In Section II, we state our assumptions about the wireless network and jamming adversary and propose evaluation metrics for flow-jamming attacks. In Section III, we formulate optimal centralized flow-jamming attacks using linear programming. In Section IV, we develop two distributed approaches for flow-jamming in the absence of a centralized adversary. In Section V, we evaluate the performance of the centralized and 
TABLE I

A SUMMARY OF NOTATION IS PROVIDED.

\begin{tabular}{c|c}
\hline Symbol & Definition \\
\hline $\mathcal{N}$ & Set of wireless network nodes \\
$\mathcal{F}$ & Collection of network flows \\
$r_{f}$ & Flow rate of flow $f \in \mathcal{F}$ \\
$\mathcal{J}$ & Set of jammers \\
$c_{j}$ & Jamming resource supply for jammer $j \in \mathcal{J}$ \\
$c_{j f}$ & Cost per unit flow rate for $j \in \mathcal{J}$ and $f \in \mathcal{F}$ \\
$x_{j f}$ & Jammer-to-flow assignment for $j \in \mathcal{J}$ and $f \in \mathcal{F}$ \\
$\mathbf{x}_{j}$ & Jammer-to-flow assignment vector for jammer $j \in \mathcal{J}$ \\
$\mathbf{x}_{f}$ & Jammer-to-flow assignment vector for flow $f \in \mathcal{F}$ \\
$\mathbf{x}$ & Jammer-to-flow assignment vector \\
$\lambda_{j}\left(\mathbf{x}_{j}\right)$ & Resource expenditure of jammer $j \in \mathcal{J}$ \\
$\Lambda(\mathbf{x})$ & Vector of resource expenditure variables \\
$I(\mathbf{x})$ & Jamming impact, see Definition 1 \\
$E(\mathbf{x})$ & Jamming efficiency, see Definition 2 \\
$V(\mathbf{x})$ & Jamming resource variation, see Definition 3 \\
\hline
\end{tabular}

distributed flow-jamming attacks using the proposed metrics. In Section VI, we summarize our results.

\section{Model Assumptions}

In this section, we state our assumptions and provide notation and definitions for the wireless network and jamming adversary. In addition, we provide metrics for the evaluation of flow-jamming attacks. A summary of notation is provided in Table I.

\section{A. Network Model}

The wireless network consists of a set of nodes $\mathcal{N}$. Data traffic between source and destination nodes in $\mathcal{N}$ is modeled by a set of single-path flows $\mathcal{F}$. We let $r_{f}$ denote the flow rate of each flow $f \in \mathcal{F}$. We assume that the nodes in $\mathcal{N}$ are fixed and the flows in $\mathcal{F}$ are fixed for the duration of the flow-jamming attack. We assume that the flows in $\mathcal{F}$ are scheduled in such a way that packet transmissions do not result in collisions, for example, by transmitting orthogonal signals in time or frequency. This assumption implies that all lost packets are the result of the flow-jamming attack and not of collisions at the MAC layer.

\section{B. Adversarial Model}

We let $\mathcal{J}$ denote the set of jammers deployed throughout the wireless network. We assume that each jammer is constrained by a finite energy supply and can jam a given packet with minimum energy expenditure by appropriately adjusting its transmission power or waveform. The minimum transmission power required to jam a packet can be computed as a function of the Jamming-power to Signal-power Ratio (JSR) [2], yielding the required power to increase the bit-error rate to a sufficient threshold. The JSR is computed as a function of the transmitter and jammer power, the network topology, and the antenna properties of each node and jammer. For simplicity,

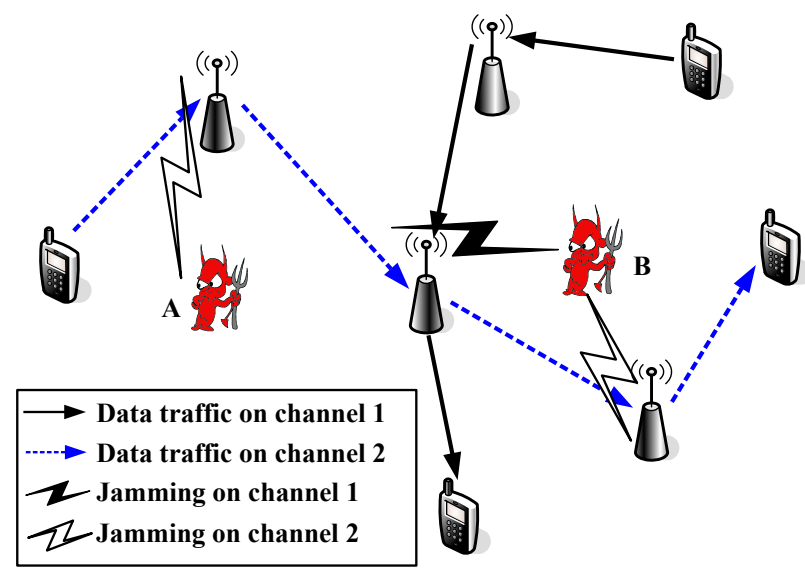

Fig. 1. A sample assignment of flow to jammers is illustrated for two network flows. In this example, jammer B completely jams the flow on channel 1 , and jammers A and B collaboratively jam the flow on channel 2 by jamming packets at corresponding receivers requiring minimum resource expenditure.

we ignore the randomness in channel variation and assume that the jamming power computed using the JSR is sufficient to jam each packet with probability one.

Each jammer $j \in \mathcal{J}$ can thus compute the JSR for each packet transmission along a flow $f$ and determine the receiving node at which the packet can be jammed with minimum resource expenditure. We let $c_{j f}$ denote the associated resource cost per unit flow rate to jam flow $f$, yielding a total resource cost of $c_{j f} r_{f}$ for jammer $j$ to jam every packet in flow $f$. However, since it is not necessary for a single jammer $j$ to jam every packet in a flow $f$, we define the jammer-to-flow assignment $x_{j f} \in[0,1]$ as the fraction of packets in flow $f$ assigned to jammer $j$. A sample assignment of flows to jammers is illustrated in Fig. 1. We denote the vector of jammer-to-flow assignment variables $x_{j f}$ for all jammers $j$ and flows $f$ by $\mathbf{x}$, for a single jammer $j$ by $\mathbf{x}_{j}$ and for a single flow $f$ by $\mathbf{x}_{f}$. Letting $c_{j}$ denote the total resource availability for jammer $j$, we define the resource expenditure $\lambda_{j}\left(\mathbf{x}_{j}\right)$ as the fraction

$$
\lambda_{j}\left(\mathbf{x}_{j}\right)=c_{j}^{-1} \sum_{f \in \mathcal{F}} c_{j f} r_{f} x_{j f}
$$

of total resources exhausted in the flow-jamming attack. We let $\Lambda(\mathbf{x})$ denote the vector of resource expenditure variables for $j \in \mathcal{J}$. For a given set of costs $c_{j f}$ and rates $r_{f}$, the flow-jamming attack is uniquely specified by the vector $\mathbf{x}$ and the schedule of specific packets to be jammed by individual jammers. By assumption, the jamming schedule has little effect on the impact of the flow-jamming attack, as packets are assumed to be transmitted without collisions. We note that it may be possible for a single jammer to simultaneously jam multiple packets transmitted over non-colliding links with a single jamming transmission. In this work, we assume such an event occurs with negligible probability, leaving the extension for future work.

We note that if the jammers in $\mathcal{J}$ are controlled by a centralized adversary, the adversary can leverage the costs 
$c_{j f}$ and rates $r_{f}$ for all jammers $j \in \mathcal{J}$ and flows $f \in \mathcal{F}$ to optimize the jammer-to-flow assignment $\mathbf{x}$ as a resource allocation problem [9]. However, if the set of jammers $\mathcal{J}$ operates with no centralized control, each jammer $j \in \mathcal{J}$ must compute the corresponding jammer-to-flow assignment $\mathbf{x}_{j}$ using a distributed protocol based on local information. These attack formulations are respectively addressed in Sections III and IV for various evaluation metrics.

\section{Evaluation Metrics}

We define metrics to evaluate the effect of a flow-jamming attack on traffic flows and the resource expenditure of the jammers. We let $\|\cdot\|_{1}$ denote the $\ell_{1}$ vector sum norm [10].

Definition 1: The jamming impact $I(\mathbf{x})$ of a flow-jamming attack with jammer-to-flow assignment $\mathbf{x}$ is defined as the average fraction of jammed flow rate over all flows in $\mathcal{F}$, given by

$$
I(\mathbf{x})=|\mathcal{F}|^{-1}\|\mathbf{x}\|_{1} .
$$

The metric of jamming impact reflects the overall effect of the flow-jamming attack on the network flows in $\mathcal{F}$ and can be used to determine the worst-case flow-jamming attack on the network flows.

Definition 2: The jamming efficiency $E(\mathbf{x})$ of a flowjamming attack with jammer-to-flow assignment $\mathbf{x}$ is defined as the ratio of jamming impact to average resource expenditure, given by

$$
E(\mathbf{x})=\frac{|\mathcal{F}|^{-1}\|\mathbf{x}\|_{1}}{|\mathcal{J}|^{-1}\|\Lambda(\mathbf{x})\|_{1}} .
$$

The metric of jamming efficiency relates the effect of the flow-jamming attack on the network flows to the resource expenditure of the jammers. We also note that the ratio $I(\mathbf{x}) / E(\mathbf{x})$ is the average resource expenditure, so this metric is expressible using those already defined. The normalization in Definition 2 thus allows for the following interpretation, independent of the number of flows $|\mathcal{F}|$ and jammers $|\mathcal{J}|$. If a jamming impact of $I(\mathbf{x})=1$ is achieved with $E(\mathbf{x}) \geq 1$, the jammers in $\mathcal{J}$ are able to completely jam the flows in $\mathcal{F}$ using a fraction $E(\mathbf{x})^{-1}$ of the available resources. If a jamming impact of $I(\mathbf{x})<1$ is achieved with $E(\mathbf{x})=1$, the jammers in $\mathcal{J}$ exhaust the maximum available resources in order to jam an average fraction $I(\mathbf{x})$ of each flow.

Definition 3: The jamming resource variation $V(\mathbf{x})$ of a flow-jamming attack with jammer-to-flow assignment $\mathbf{x}$ is defined as the relative difference between the maximum and minimum resource expenditure, given by

$$
V(\mathbf{x})=1-\frac{\min _{j} \Lambda(\mathbf{x})}{\max _{j} \Lambda(\mathbf{x})} .
$$

The jamming resource variation measures the balance in resource expenditure over the set of jammers. If the resource variation is large, i.e. near 1 , some jammers will fully exhaust their battery energy and be unable to participate in the flowjamming attack, thus degrading the lifetime of the attack. If, on the other hand, the resource variation is small, i.e. near 0 , then the minimum jammer lifetime will be maximized, thus prolonging the duration of the flow-jamming attack.

\section{Centralized Flow-Jamming Attacks}

In this section, we formulate flow-jamming attacks which are optimal with respect to the evaluation metrics proposed in Section II-C. We first present the maximum impact flowjamming attack, using the jamming impact $I(\mathbf{x})$ as the primary optimization metric and the jamming efficiency $E(\mathbf{x})$ as a secondary optimization metric. We next present the efficient flow-jamming attack, using the jamming efficiency as the primary optimization metric. We then present the balanced flow-jamming attack, using the jamming resource variation $V(\mathbf{x})$ as the primary optimization metric and the jamming impact $I(\mathbf{x})$ as the secondary optimization metric. Each flowjamming attack is formulated as an optimization problem, and each is solved using linear programming techniques.

The jammer-to-flow assignment $\mathbf{x}$ corresponding to any flow-jamming attack must satisfy the following constraints. The resource expenditure $\lambda_{j}\left(\mathbf{x}_{j}\right)$ for each jammer $j \in \mathcal{J}$, as defined in (1), must satisfy the supply constraint

$$
\lambda_{j}\left(\mathbf{x}_{j}\right) \leq 1,
$$

as each jammer cannot exhaust more than the available resources. The assignment of jammers to each flow $f \in \mathcal{F}$ must additionally satisfy the flow constraint

$$
\left\|\mathbf{x}_{f}\right\|_{1} \leq 1,
$$

as the jammers cannot jam more flow than is present.

\section{A. Maximum Impact Flow-Jamming Attacks}

The maximum impact flow-jamming attack primarily maximizes the jamming impact $I(\mathbf{x})$ and secondarily maximizes the jamming efficiency $E(\mathbf{x})$. We develop an algorithm for maximum impact flow-jamming attacks by deriving a linear program corresponding to each of two cases: $I(\mathbf{x})=1$ and $I(\mathbf{x})<1$.

The case of $I(\mathbf{x})=1$ corresponds to the ability to achieve equality in the flow constraint in (3) for all $f \in \mathcal{F}$. If this condition can be achieved for the given resource supply variables $c_{j}$ for $j \in \mathcal{J}$ and network and jammer topology, the jamming efficiency is maximized by minimizing the total resource expenditure $\|\Lambda(\mathbf{x})\|_{1}$ subject to the supply constraint in (2). The formulation of this flow-jamming attack is stated in Fig. 2(a) as a linear program. We note that the flow-jamming attack in this case is representative of the Hitchcock problem [9] for minimum cost resource allocation, where the flow constraint is interpreted as a resource demand.

The case of $I(\mathbf{x})<1$ corresponds to the inability to achieve equality in the flow constraint in (3) for all $f \in \mathcal{F}$. In this case, we note that each jammer $j \in \mathcal{J}$ will contribute as much of the available resource supply as possible to the subset of $\mathcal{F}$ of flows $f$ with cost $c_{j f}<\infty$. Hence, the jamming impact and efficiency are simultaneously maximized by maximizing the total fraction of jammed flow rate $\|\mathbf{x}\|_{1}$ subject to the supply constraint in (2) and the flow constraint in (3). The formulation of this flow-jamming attack is stated in Fig. 2(b) as a linear program. 


\begin{aligned} & \hline $\min \|\Lambda(\mathbf{x})\|_{1} \\ &$ s.t. $\lambda_{j}\left(\mathbf{x}_{j}\right) \leq 1$ for all $j \in \mathcal{J} \\ &,\left\|\mathbf{x}_{f}\right\|_{1}=1$ for all $f \in \mathcal{F} \\ &, 0 \leq x_{j f} \leq 1$ for all $j \in \mathcal{J}, f \in \mathcal{F} \\ &$. \hline\end{aligned}

(a)

\begin{aligned} & \hline $\max \|\mathbf{x}\|_{1} \\ &$ s.t. $\lambda_{j}\left(\mathbf{x}_{j}\right) \leq 1$ for all $j \in \mathcal{J} \\ &\left\|\mathbf{x}_{f}\right\|_{1} \leq 1$ for all $f \in \mathcal{F} \\ &, 0 \leq x_{j f} \leq 1$ for all $j \in \mathcal{J}, f \in \mathcal{F} \\ &$. \hline\end{aligned}

(b)

Fig. 2. The maximum impact flow-jamming attack algorithm first attempts to solve the linear program in (a) with equality in the flow constraint (3). If no solution is feasible, the linear program in (b) is solved.

The combination of the linear programs in Fig. 2 yields the desired centralized algorithm for maximum impact flowjamming attacks. The first step of the algorithm is to attempt to solve the linear program in Fig. 2(a), yielding the jammer-to-flow assignment vector $\mathbf{x}$ which maximizes $E(\mathbf{x})$ for $I(\mathbf{x})=1$. If a feasible solution to the first linear program does not exist, a solution to the linear program in Fig. 2(b) is computed. Since the algorithm involves computing solutions to linear programs, it runs in polynomial time [9]. Furthermore, a feasible solution is guaranteed, as the trivial solution $\mathbf{x}=0$ is feasible for the linear program in Fig. 2(b).

\section{B. Efficient Flow-Jamming Attacks}

The efficient flow-jamming attack aims to maximize the jamming efficiency $E(\mathbf{x})$ subject to the supply constraint in (2) and the flow constraint in (3). The optimization problem is formulated in Fig. 3(a). However, since $E(\mathbf{x})$ is not a linear function in the jammer-to-flow assignment variables, we provide a linear approximation in Fig. 3(b) which approximates the optimal solution of the problem in Fig. 3(a) using a linear objective function. The following result proves that the linear approximation is tight.

Theorem 1: Let $\mathrm{x}^{*}$ denote the non-zero jammer-to-flow assignment which maximizes $E(\mathbf{x})$ and $\hat{\mathbf{x}}$ denote the non-zero jammer-to-flow assignment which minimizes $|\mathcal{J}|^{-1}\|\Lambda(\mathbf{x})\|_{1}-$ $\epsilon^{-1}|\mathcal{F}|^{-1}\|\mathbf{x}\|_{1}$ for a given $\epsilon>0$. Then $0 \leq E\left(\mathbf{x}^{*}\right)-E(\hat{\mathbf{x}}) \leq \epsilon$.

Proof: Let $g(\mathbf{x})=|\mathcal{J}|^{-1}\|\Lambda(\mathbf{x})\|_{1}$ and $\ell(\mathbf{x})=|\mathcal{F}|^{-1}\|\mathbf{x}\|_{1}$ such that $E(\mathbf{x})=\ell(\mathbf{x}) / g(\mathbf{x})$ and $\mathbf{x}^{*}$ is given by

$$
\begin{aligned}
\mathbf{x}^{*} & =\underset{\mathbf{x}>0}{\arg \max } E(\mathbf{x})=\underset{\mathbf{x}>0}{\arg \max } \frac{\ell(\mathbf{x})}{g(\mathbf{x})} \\
& =\underset{\mathbf{x}>0}{\arg \min } \frac{g(\mathbf{x})}{\ell(\mathbf{x})}=\underset{\mathbf{x}>0}{\arg \min }\left(\epsilon \frac{g(\mathbf{x})}{\ell(\mathbf{x})}-1\right),
\end{aligned}
$$

where the final equality in (4) holds because the optimal solution is not changed by an affine transformation of the

\begin{aligned} & \hline $\max \frac{|\mathcal{F}|^{-1}\|\mathbf{x}\|_{1}}{|\mathcal{J}|^{-1}\|\Lambda(\mathbf{x})\|_{1}} \\ &$ s.t. $\lambda_{j}\left(\mathbf{x}_{j}\right) \leq 1$ for all $j \in \mathcal{J} \\ &,\left\|\mathbf{x}_{f}\right\|_{1} \leq 1$ for all $f \in \mathcal{F} \\ &, 0 \leq x_{j f} \leq 1$ for all $j \in \mathcal{J}, f \in \mathcal{F} \\ &$. \hline\end{aligned}

(a)

\begin{aligned} & \hline $\min |\mathcal{J}|^{-1}\|\Lambda(\mathbf{x})\|_{1}-\epsilon^{-1}|\mathcal{F}|^{-1}\|\mathbf{x}\|_{1} \\ &$ s.t. $\lambda_{j}\left(\mathbf{x}_{j}\right) \leq 1$ for all $j \in \mathcal{J} \\ &,\left\|\mathbf{x}_{f}\right\|_{1} \leq 1$ for all $f \in \mathcal{F} \\ &, 0 \leq x_{j f} \leq 1$ for all $j \in \mathcal{J}, f \in \mathcal{F} \\ &$. \hline\end{aligned}

(b)

Fig. 3. The optimal efficient flow-jamming attack can be obtained by solving the non-linear optimization problem in (a). The linear approximation in (b) yields a solution within an additive constant $\epsilon$ of the optimal solution, as given by Theorem 1 .

objective function. The solution $\hat{\mathbf{x}}$ is similarly given by

$$
\hat{\mathbf{x}}=\underset{\mathbf{x}>0}{\arg \min }\left(g(\mathbf{x})-\epsilon^{-1} \ell(\mathbf{x})\right) \text {. }
$$

Optimality of the corresponding solutions $\mathbf{x}^{*}$ and $\hat{\mathbf{x}}$ implies the inequalities

$$
\begin{aligned}
\frac{\ell\left(\mathbf{x}^{*}\right)}{g\left(\mathbf{x}^{*}\right)} & \geq \frac{\ell(\hat{\mathbf{x}})}{g(\hat{\mathbf{x}})}, \\
\frac{g\left(\mathbf{x}^{*}\right)-\epsilon^{-1} \ell\left(\mathbf{x}^{*}\right)}{\epsilon^{-1} \ell\left(\mathbf{x}^{*}\right)} & \leq \frac{g(\hat{\mathbf{x}})-\epsilon^{-1} \ell(\hat{\mathbf{x}})}{\epsilon^{-1} \ell(\hat{\mathbf{x}})}, \\
g(\hat{\mathbf{x}})-\epsilon^{-1} \ell(\hat{\mathbf{x}}) & \leq g\left(\mathbf{x}^{*}\right)-\epsilon^{-1} \ell\left(\mathbf{x}^{*}\right) .
\end{aligned}
$$

The combination of (7) and (8) yields the inequality $\ell\left(\mathbf{x}^{*}\right) \geq$ $\ell(\hat{\mathbf{x}})$. This result and (8) yield the inequality

$$
g\left(\mathbf{x}^{*}\right)-g(\hat{\mathbf{x}}) \geq \epsilon^{-1}\left(\ell\left(\mathbf{x}^{*}\right)-\ell(\hat{\mathbf{x}})\right) \geq 0 .
$$

If $g\left(\mathbf{x}^{*}\right)=g(\hat{\mathbf{x}})$ then $E\left(\mathbf{x}^{*}\right)=E(\hat{\mathbf{x}})$, so the proof holds. Hence, for the remainder of the proof, assume $g\left(\mathbf{x}^{*}\right)>g(\hat{\mathbf{x}})$.

Multiplying (6) through by $g\left(\mathbf{x}^{*}\right) g(\hat{\mathbf{x}})$, subtracting the term $\ell\left(\mathbf{x}^{*}\right) g\left(\mathbf{x}^{*}\right)$, and rearranging non-zero terms yields the inequality

$$
\frac{\ell\left(\mathbf{x}^{*}\right)}{g\left(\mathbf{x}^{*}\right)} \leq \frac{\ell\left(\mathbf{x}^{*}\right)-\ell(\hat{\mathbf{x}})}{g\left(\mathbf{x}^{*}\right)-g(\hat{\mathbf{x}})} .
$$

Combining the inequalities in (6), (9), and (10) yields

$$
\frac{\ell(\hat{\mathbf{x}})}{g(\hat{\mathbf{x}})} \leq \frac{\ell\left(\mathbf{x}^{*}\right)}{g\left(\mathbf{x}^{*}\right)} \leq \epsilon .
$$

Since both $\ell\left(\mathbf{x}^{*}\right) / g\left(\mathbf{x}^{*}\right)$ and $\ell(\hat{\mathbf{x}}) / g(\hat{\mathbf{x}})$ are positive and bounded by $\epsilon$, their difference is also bounded by $\epsilon$.

\section{Balanced Flow-Jamming Attacks}

The balanced flow-jamming attack primarily minimizes the jamming resource variation $V(\mathbf{x})$ and secondarily maximizes the jamming impact $I(\mathbf{x})$ and jamming efficiency $E(\mathbf{x})$. We 


\begin{aligned} & \hline $\min \lambda \\ &$ s.t. $\lambda_{j}\left(\mathbf{x}_{j}\right) \leq \lambda$ for all $j \in \mathcal{J} \\ &,\left\|\mathbf{x}_{f}\right\|_{1}=1$ for all $f \in \mathcal{F} \\ &, 0 \leq x_{j f} \leq 1$ for all $j \in \mathcal{J}, f \in \mathcal{F} \\ &, 0 \leq \lambda \leq 1 \\ &$. \hline\end{aligned}

(a)

\begin{aligned} & \hline $\max \lambda \\ &$ s.t. $\lambda \leq \lambda_{j}\left(\mathbf{x}_{j}\right) \leq 1$ for all $j \in \mathcal{J} \\ &,\left\|\mathbf{x}_{f}\right\|_{1} \leq 1$ for all $f \in \mathcal{F} \\ &, 0 \leq x_{j f} \leq 1$ for all $j \in \mathcal{J}, f \in \mathcal{F} \\ &, 0 \leq \lambda \leq 1 \\ &$\hline\end{aligned}

(b)

Fig. 4. The balanced flow-jamming attack algorithm first attempts to solve the linear program in (a) with equality in the flow constraint (3). If no solution is feasible, the linear program in (b) is solved.

develop an algorithm for balanced flow-jamming attacks by deriving a linear program corresponding to each of two cases: $I(\mathbf{x})=1$ and $I(\mathbf{x})<1$.

The case of $I(\mathbf{x})=1$ corresponds to the ability to achieve equality in the flow constraint in (3) for all $f \in \mathcal{F}$. If this condition can be achieved for the given resource supply variables $c_{j}$ for $j \in \mathcal{J}$ and network and jammer topology, the jamming resource variation is minimized with maximum jamming impact by minimizing the variable $\lambda=\max _{j} \Lambda(\mathrm{x})$ subject to the supply constraint in (2). In this case, minimizing $\lambda$ corresponds to maximizing the jamming efficiency $E(\mathbf{x})$. This flow-jamming attack can be formulated as a linear program by aiming to maximize $\lambda$ with the additional constraint $\lambda_{j}\left(\mathbf{x}_{j}\right) \leq \lambda$ for all $j \in \mathcal{J}$, a stronger constraint than the supply constraint in (2). The formulation of this flow-jamming attack is stated in Fig. 4(a) as a linear program.

The case of $I(\mathbf{x})<1$ corresponds to the inability to achieve equality in the flow constraint in (3) for all $f \in \mathcal{F}$. In this case, the resource variation $V(\mathbf{x})$ is minimized with maximum jamming impact $I(\mathbf{x})$ and efficiency $E(\mathbf{x})$ by maximizing $\lambda=\min _{j} \Lambda(\mathbf{x})$ subject to the supply constraint in (2) for all $j \in \mathcal{J}$ and the flow constraint in (3) for all $f \in \mathcal{F}$. This flow-jamming attack can be formulated as a linear program by maximizing $\lambda$ subject to the additional constraint that $\lambda \leq \lambda_{j}\left(\mathbf{x}_{j}\right)$, introducing a lower bound into the supply constraint in (2) for each $j \in \mathcal{J}$. The formulation of this flow-jamming attack is stated in Fig. 4(b) as a linear program.

The combination of the linear programs in Fig. 4 yields the desired centralized algorithm for balanced flow-jamming attacks. The centralized algorithm is obtained using a similar technique to that in Section III-A, first attempting to solve the linear program in Fig. 4(a), solving that in Fig. 4(b) if no solution is feasible. As previously discussed, the algorithm runs in polynomial time and is guaranteed to have a feasible solution.

\section{Distributed Flow-Jamming Attacks}

In this section, we investigate distributed algorithms for flow-jamming attacks in which each jammer $j$ uses only local information to compute the jammer-to-flow assignment $\mathbf{x}_{j}$. We assume that each jammer $j$ exchanges information with a subset $\mathcal{J}_{j} \subseteq \mathcal{J}$ of neighboring jammers (with $j \in \mathcal{J}_{j}$ itself) and has knowledge of the subset $\mathcal{F}_{j} \subseteq \mathcal{F}$ of flows for which $c_{j f}<\infty$. We assume the variables $c_{j f} / c_{j}$ are distinct for all $j \in \mathcal{J}$ and $f \in \mathcal{F}$, noting that this assertion holds with probability 1 if there is any source of randomness in $c_{j f}$.

We propose two approaches for distributed flow-jamming attacks with contrasting characteristics. The first approach uses linear programming techniques similar to the centralized approach in Section III for an aggressive flow-jamming attack, seeking the maximal jamming impact in trade for a decrease in jamming efficiency $E(\mathbf{x})$. The second approach yields an attack algorithm for flow-jamming attacks that are conservative in terms of resource expenditure, seeking the maximal jamming efficiency $E(\mathbf{x})$ in trade for a decrease in jamming impact $I(\mathbf{x})$. In what follows, we describe the two distributed flow-jamming attacks and discuss trade-offs between the two approaches.

\section{A. Local Linear Programming for Maximum Jamming Impact}

The first approach for distributed flow-jamming attacks is obtained by allowing each jammer $j$ to solve a linear program to obtain the jammer-to-flow assignment $\mathbf{x}_{j}$ using only local information. By replacing the sets $\mathcal{J}$ and $\mathcal{F}$ in each linear program in Section III with the corresponding locally available sets $\mathcal{J}_{j}$ and $\mathcal{F}_{j}$, respectively, each jammers $j$ can locally compute a jammer-to-flow assignment $\mathbf{x}$, keeping only the corresponding assignment vector $\mathbf{x}_{j}$. Hence, when each jammer's local neighborhood is expanded to include the entire sets $\mathcal{J}$ and $\mathcal{F}$, the optimal solutions to the attacks illustrated in Section III are achieved. However, when $\left|\mathcal{J}_{j}\right|<|\mathcal{J}|$, the local information available to jammer $j$ may be different from neighboring jammers, and the computed solutions may not agree, leading to sub-optimal flow-jamming attacks.

Using a local variant of either the maximum impact attack in Section III-A or the efficient attack in Section III-B will likely achieve a near-optimal jamming impact $I(\mathbf{x})$. However, due to the lack of global information in the local linear programming approach, it is likely that distant jammers will over-provision resources to a single flow, corresponding to a violation of the constraint in (3). This over-provisioning does not significantly reduce the jamming impact, but it significantly increases the total resource expenditure, leading to a decreased jamming efficiency.

The nature of this distributed flow-jamming attack using local linear programming suggests that it is a viable solution when the jammers have excess jamming resources and can afford to over-provision the available resources for the sake 
of jamming impact. However, when the jammers are severely resource constrained, this luxury may not be affordable, and an alternate solution is required.

\section{B. Heuristic Algorithm for Efficient Flow-Jamming}

The second approach for distributed flow-jamming attacks uses a conservative heuristic for efficient flow-jamming based on the following observations. First, given two flows $f_{1}$ and $f_{2}$ and a single jammer $j$, the jamming efficiency is maximized by assigning resources to the flow with lower normalized cost $c_{j f} / c_{j}$ before assigning resources to the other flow. This allows the jammer $j$ to maximize the jammed flow rate at minimum resource expenditure. Second, given two jammers $j_{1}$ and $j_{2}$ and a single flow $f$, the jamming efficiency is maximized by assigning resources to the jammer with lower normalized cost $c_{j f} / c_{j}$ before assigning flow to the other jammer.

By applying this conservative heuristic to an arbitrary number of flows and jammers at a given instant during attack execution, each jammer $j$ considers only the single flow $f^{*}$ with minimum normalized cost $c_{j f^{*}} / c_{j}$ and only assigns resources to $f^{*}$ if there is no neighboring jammer $j^{\prime} \in \mathcal{J}_{j}$ for which $c_{j^{\prime} f^{*}} / c_{j^{\prime}}<c_{j f^{*}} / c_{j}$. This heuristic implicitly constructs a strict partial ordering [11] on the set $\mathcal{J} \times \mathcal{F}$ of ordered jammer-flow pairs $(j, f)$ and use this ordering to compute jammer-to-flow assignments. In a local neighborhood, each jammer $j \in \mathcal{J}$ effectively follows a sub-ordering on $\mathcal{J}_{j} \times \mathcal{F}_{j}$ and assigns resources to flows according to the relations imposed by the sub-ordering.

To perform the heuristic algorithm, each jammer $j$ must know the normalized costs $c_{j^{\prime} f} / c_{j^{\prime}}$ for each neighboring jammer $j^{\prime} \in \mathcal{J}_{j}$ and flow $f \in \mathcal{F}_{j}$. The normalized costs are constant, but can be updated to $\infty$ for any neighboring jammer that will not contribute further to a flow. Furthermore, in assigning resources to a flow $f$, jammer $j$ must know the fraction $y_{f}$ of flow which is already assigned to neighboring jammers $j^{\prime} \in \mathcal{J}_{j}$. The variables $y_{f}$ must be updated during the attack to reflect the progressive assignment of flow to neighboring jammers. Similarly, jammer $j$ must notify the neighboring jammers when each jammer-to-flow assignment variable $x_{j f}$ is determined and when its resources have been exhausted. The heuristic flow-jamming attack algorithm is presented in its entirety in Fig. 5.

If the flow $f^{*}$ with minimum normalized cost for jammer $j$ can be assigned to a neighboring jammer with lower normalized cost, jammer $j$ is required to wait for a notification. If every jammer in $\mathcal{J}$ is simultaneously waiting for notifications, however, the algorithm will stall indefinitely. Termination of the heuristic algorithm in finite time is guaranteed by the following result.

Theorem 2: The heuristic algorithm for distributed flowjamming attacks in Fig. 5 terminates in finite time for all jammers $j \in \mathcal{J}$.

Proof: The algorithm stalls indefinitely if every jammer is simultaneously waiting for notifications. If a single jammer is not waiting, the resulting notification will allow neighboring

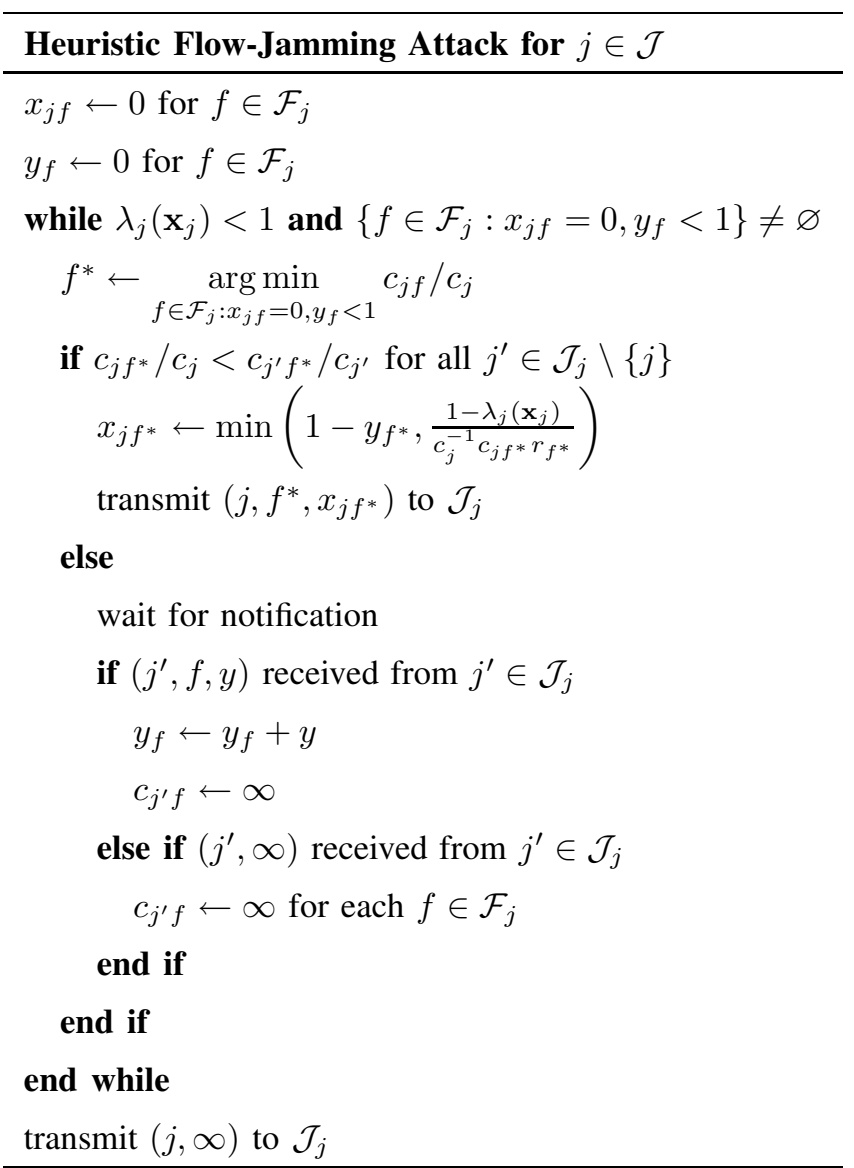

Fig. 5. This heuristic algorithm attempts to maximize the jamming efficiency and the jamming impact, similar to the centralized efficient flow-jamming attack in Section III-B.

jammers to progress. We thus show that there is always at least one jammer that is not waiting. We prove the desired result by constructing a directed graph $G=(V, E)$ corresponding to the strict partial ordering discussed above. The vertex set $V$ of $G$ is given by the set $\mathcal{J} \times \mathcal{F}$. A directed edge $\left(\left(j_{1}, f_{1}\right),\left(j_{2}, f_{2}\right)\right)$ is in the edge set $E$ of $G$ if and only if $c_{j_{1} f_{1}} / c_{j_{1}}<c_{j_{2} f_{2}} / c_{j_{2}}$ and either $f_{1}=f_{2}$ or $j_{1}=j_{2}$. Since it is constructed from the strict partial ordering, $G$ is an acyclic graph [11], as otherwise a cycle traversing the vertex $\left(j_{1}, f_{1}\right)$ in $G$ would represent a sequence of strict inequalities beginning and ending with $c_{j_{1} f_{1}} / c_{j_{1}}$. Since $G$ is a directed acyclic graph, there must be at least one vertex $\left(j^{*}, f^{*}\right) \in V$ with no incoming edge [11]. By construction of the partial ordering, jammer $j^{*}$ will not wait for any other jammer to compute the jammer-to-flow assignment variable $x_{j^{*} f^{*}}$ for flow $f^{*}$. As the algorithm progresses and variables $x_{j f}$ are computed, the corresponding vertices $(j, f)$ are removed from $G$. Finally, since any subgraph of a directed acyclic graph is a directed acyclic graph, there always exists such a vertex $\left(j^{*}, f^{*}\right)$.

We note that the algorithm presented in this section assumes that each jammer $j$ knows the flow rate $r_{f}$ of each flow $f \in \mathcal{F}_{j}$. However, these parameters may not be available to the jammer $j$, especially if jammers are not exchanging information between neighborhoods. Hence, in this case, each jammer 
$j$ must compute an estimate $r_{j f}^{\prime}$ of the residual throughput that has not been jammed by upstream jammers. Moreover, the algorithm does not account for over-provisioning of jamming resources between distant neighborhoods in the jammer network, so basing the attack on the residual flow $r_{j f}^{\prime}$ and updating this quantity over time may reduce over-provisioning and reduce jamming resource expenditure. However, such an approach assigns a higher fraction of flow rate to jammers near the flow sources.

The nature of the heuristic flow-jamming attack using local ordering leads to a solution with high jamming efficiency, as no effort is taken to jam flows that are quite costly unless there are excess jamming resources. However, since these costly flows are initially ignored, the corresponding jamming impact may be significantly reduced. Hence, this approach is may be quite effective when the jammers are severely resource constrained.

\section{Performance Evaluation}

In this section, we evaluate and compare the jamming impact, efficiency, and resource variation for various flowjamming attacks. We first evaluate the performance of the centralized maximum impact, efficient, and balanced flowjamming attacks using the linear program formulations in Section III. We then evaluate the performance of the two distributed flow-jamming attacks proposed in Section IV.

In our simulation study, we consider a network of $|\mathcal{N}|$ randomly deployed nodes with $|\mathcal{F}|$ shortest-path flows between randomly selected source and destination nodes. The jammers in $\mathcal{J}$ are randomly deployed, and each cost $c_{j f}$ is proportional to the minimum squared distance from jammer $j$ to any non-source node participating in the flow $f$. The cost $c_{j f}$ is infinite if the minimum distance from jammer $j$ to flow $f$ is greater than a fixed range. The total jamming resource $\sum_{j \in \mathcal{J}} c_{j}$ is equally distributed among the jammers, effectively distributing the available jamming energy more evenly over the network as $|\mathcal{J}|$ increases. We note that random node deployment, random source and destination selection, and random jammer deployment suggest that the variance among individual simulated instances is relatively high. Hence, each plotted curve illustrates an average over 100 simulated instances to capture the average performance of the flowjamming attacks. Note that the network size $|\mathcal{N}|$ is fixed at 200 nodes for all simulation runs, as the effect of flow-jamming attacks is influenced by a large number of network and routing protocol parameters that have been abstracted in this study due to space constraints.

\section{A. Evaluation of Centralized Attacks}

We simulate the three centralized flow-jamming attacks to compare the metrics of jamming impact, efficiency, and resource variation as a function of various parameters. For each of the centralized attacks, we perform a number of individual simulations which investigate the effect of individual parameters on the performance of flow-jamming attacks. We vary the number of jammers $|\mathcal{J}|$, the total jamming resources $\sum_{j \in \mathcal{J}} c_{j}$, and the number of network flows $|\mathcal{F}|$. Intuitively, we expect the jamming impact to be greatest for the maximum impact attack, followed by that of the efficient attack and the balanced attack. This is due to the fact that jamming impact is a secondary metric for the efficient and balanced attacks, and the balanced attack effectively imposes an additional constraint on the efficient attack. Similarly, we expect the jamming efficiency to be greatest for the efficient attack, followed by that of the maximum impact attack and the balanced attack. Finally, we expect the jamming resource variation to be least for the balanced attack, followed by the two remaining attacks, leading to the added benefit of a longer jammer lifetime under the balanced flow-jamming attack.

Fig. 6 illustrates the jamming impact, efficiency, and resource variation resulting from the centralized flow-jamming attack formulations as a function of the number of jammers $|\mathcal{J}|$ participating in the flow-jamming attack. In this simulation, the number of flows $|\mathcal{F}|$ is fixed at 50 and the total jamming resource $\sum_{j \in \mathcal{J}} c_{j}$ is fixed at a normalized value of 1. From Fig. 6(a) and Fig. 6(b), we see that the impact and efficiency of the attacks improve as the number of jammers $|\mathcal{J}|$ is increased. Similarly, from Fig. 6(c), we see that the resource variation degrades with the number of jammers $|\mathcal{J}|$. These changes are due to the higher density of jammers through the network, implying that the average cost $c_{j f}$ to jam each flow is decreased and the probability that at least one jammer will have a relatively lower cost is increased.

Fig. 7 illustrates the jamming impact, efficiency, and resource variation resulting from the centralized flow-jamming attack formulations as a function of the available jamming resources $\sum_{j \in \mathcal{J}} c_{j}$, normalized with respect to the fixed value in Fig. 6. In this simulation, the number of flows $|\mathcal{F}|$ is fixed at 50 and the number of jammers $|\mathcal{J}|$ is fixed at 10 . From Fig. 7(a) and Fig. 7(b), we see that the impact and efficiency of the attacks improve as the jamming resource increases. Similarly, from Fig. 7(c), we see that the resource variation degrades with the available jamming resources. These changes are due to increased impact of the flow-jamming attacks due to the single jammer $j \in \mathcal{J}$ with the lowest cost $c_{j f}$ for each flow.

Fig. 8 illustrates the jamming impact, efficiency, and resource variation resulting from the centralized flow-jamming attack formulations as a function of the number of network flows $|\mathcal{F}|$. In this simulation, the number of jammers $|\mathcal{J}|$ is fixed at 10 , and the total jamming resource $\sum_{j \in \mathcal{J}} c_{j}$ is fixed at the normalized value 1. From Fig. 8(a), we see that the impact of the flow-jamming attacks does not vary much, as the jammers have sufficient resources to jam whichever flows are nearby. However, from Fig. 8(b), we see that efficiency degrades significantly as the number of flows increases, as each jammer is required to exhaust a significantly larger fraction of the available resources to jam the nearby flows. Fig. 8(c) illustrates the corresponding improvement in resource variation due to the fact that each jammer is becoming closer to exhausting the available resources as the number of nearby flows increases. 


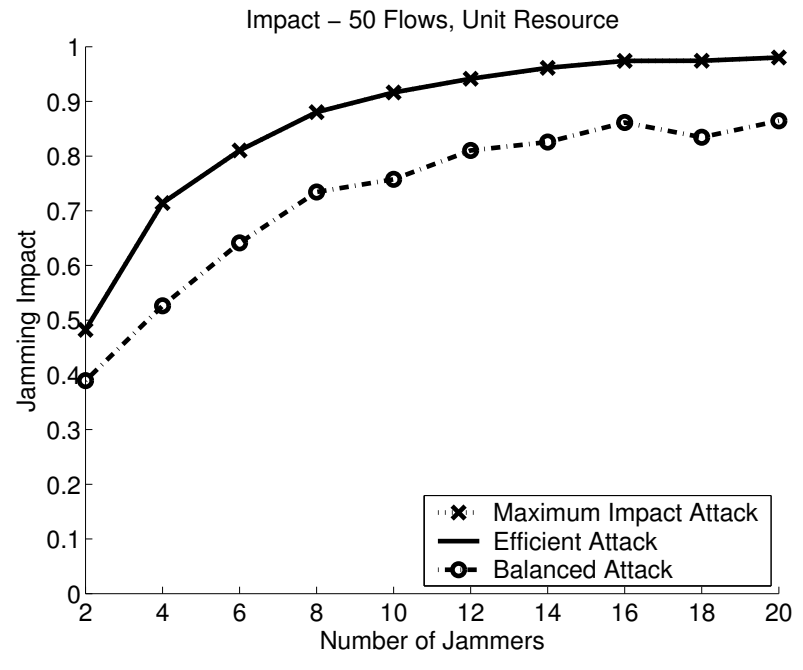

(a)

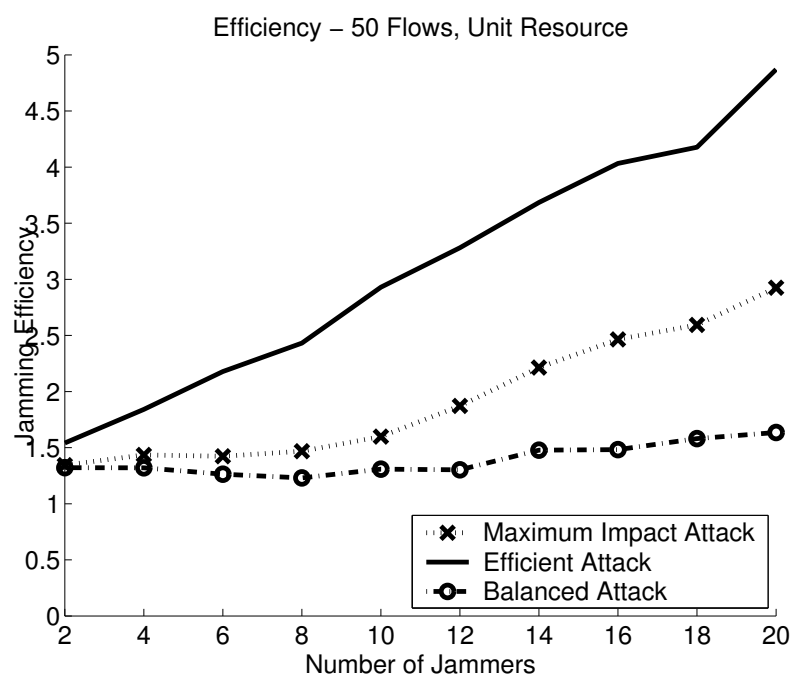

(b)

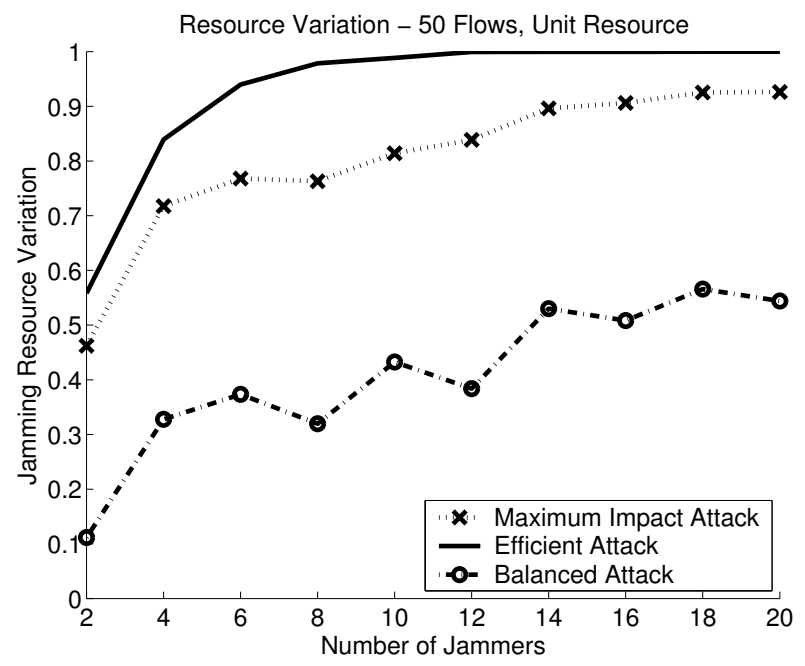

(c)

Fig. 6. We compare the maximum impact, efficient, and balanced flowjamming attacks in Section III in terms of the (a) jamming impact, (b) jamming efficiency, and (c) jamming resource variation when the number of jammers $|\mathcal{J}|$ is varied.

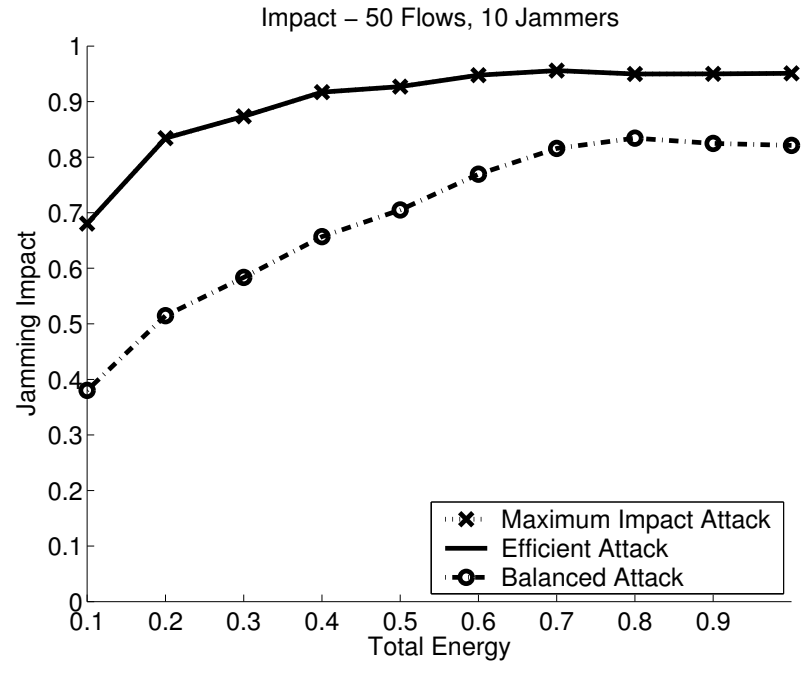

(a)

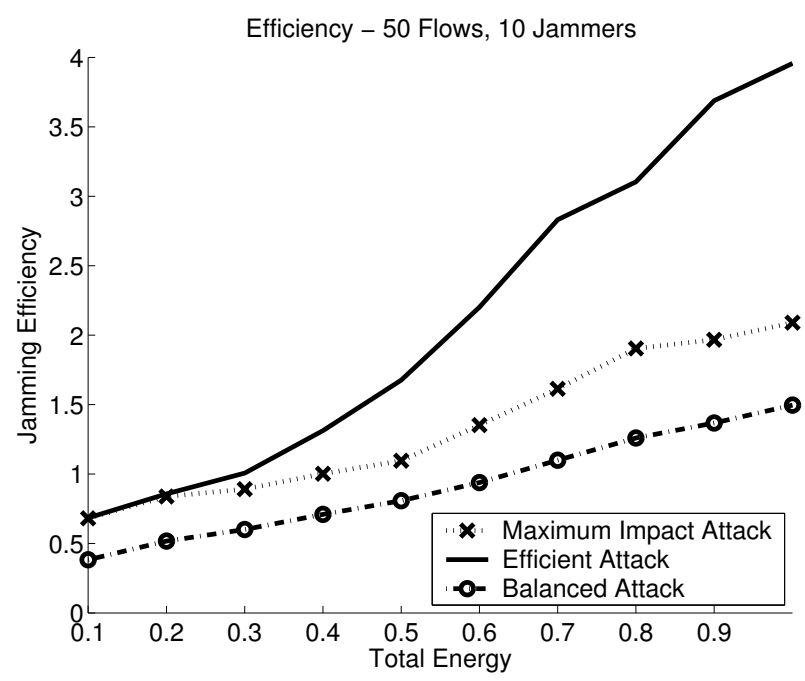

(b)

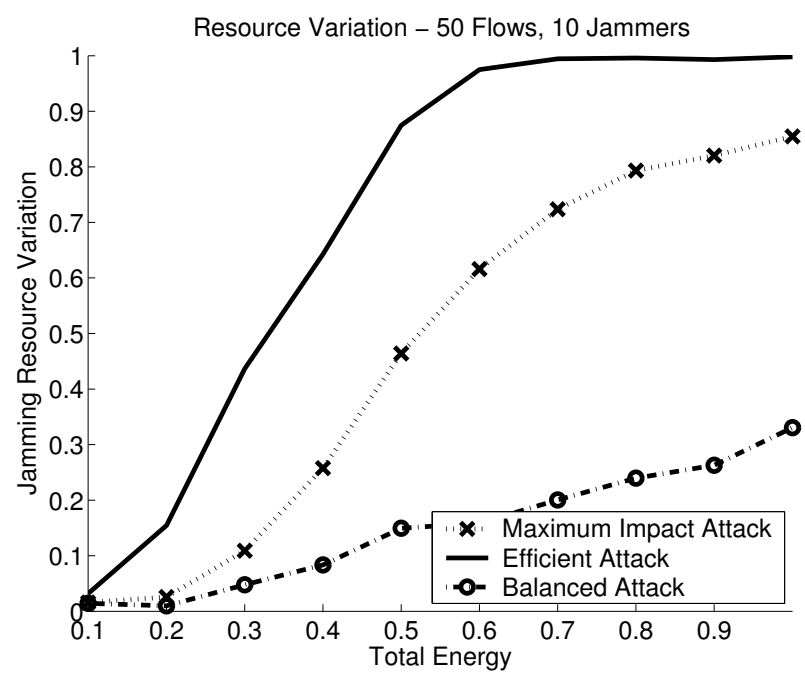

(c)

Fig. 7. We compare the maximum impact, efficient, and balanced flowjamming attacks in Section III in terms of the (a) jamming impact, (b) jamming efficiency, and (c) jamming resource variation when the total jamming resource is varied. 


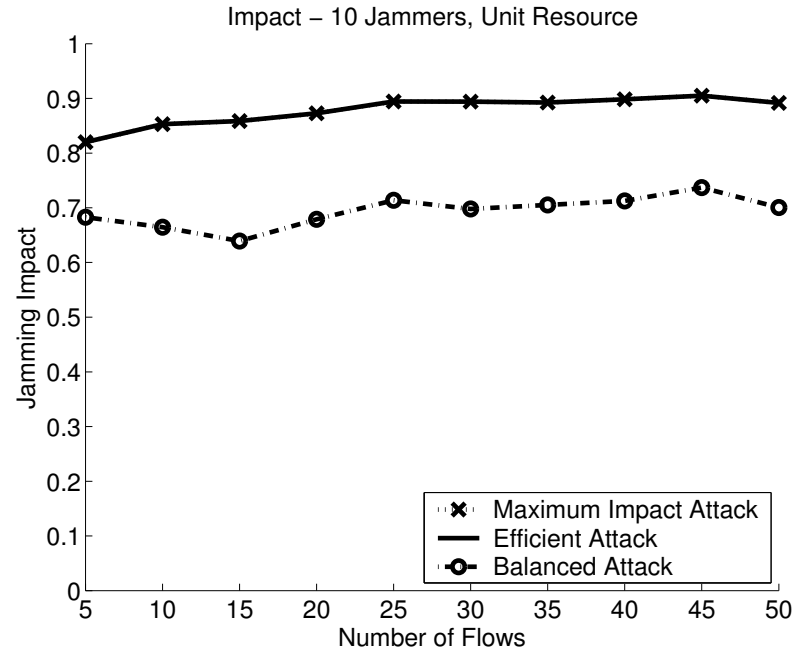

(a)

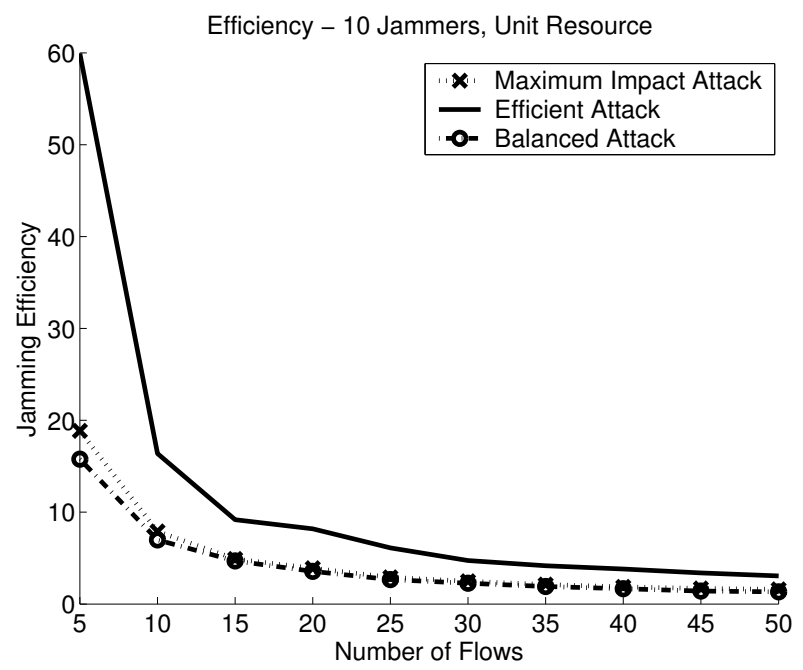

(b)

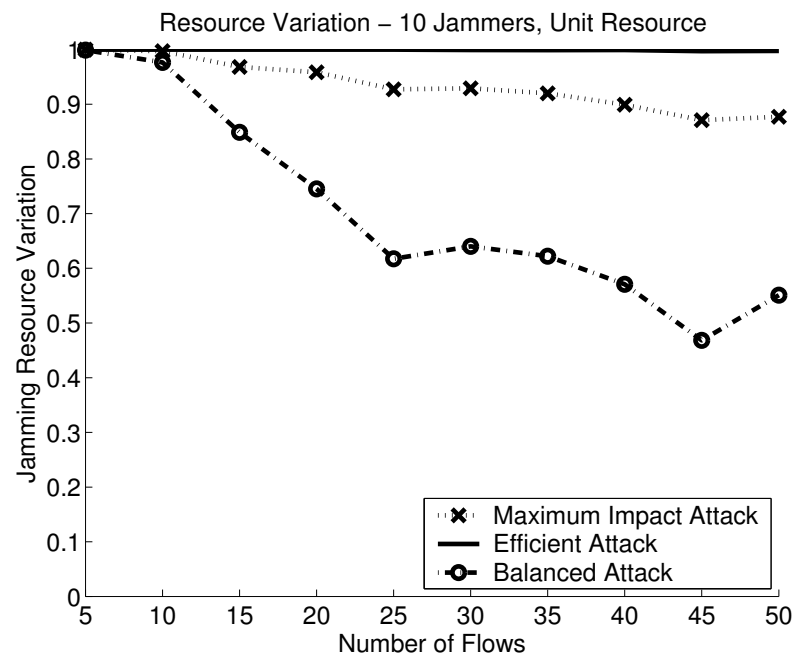

(c)

Fig. 8. We compare the maximum impact, efficient, and balanced flowjamming attacks in Section III in terms of the (a) jamming impact, (b) jamming efficiency, and (c) jamming resource variation when the number of network flows is varied.

\section{B. Evaluation of Distributed Attacks}

We simulate the two types of distributed flow-jamming attacks proposed in Section IV to compare the effect of the attacks with respect to the proposed metrics as a function of the average neighborhood size $\left|\mathcal{J}_{j}\right|$ of each jammer $j$. Due to the distributed nature of the attacks, we expect the jamming resource variation $V(\mathbf{x})$ to be quite high, so we only address the metrics of jamming impact and efficiency and perform only the distributed equivalents of the maximum impact and efficient flow-jamming attacks. Due to space constraints, we evaluate the impact and efficiency as a function of the number of jammers $|\mathcal{J}|$ and the average neighborhood size, as a percentage of the jammers $|\mathcal{J}|$. We expect the behavior with respect to other parameter variations to correspondingly mirror the variation seen here and under the centralized attacks.

Fig. 9 illustrates the jamming impact and efficiency resulting from the distributed version of the efficient flow-jamming attack using the local linear programming approach as a function of the number of jammers $|\mathcal{J}|$. In this simulation, the number of flows $|\mathcal{F}|$ is fixed at 50 , and the total jamming resource $\sum_{j \in \mathcal{J}} c_{j}$ is fixed at the normalized value 1 . From Fig. 9(a), we see that the impact of the distributed flowjamming attack is nearly as high as that of the centralized efficient attack (when the neighborhood size contains all of the jammers). From Fig. 9(b), we see that the efficiency of the distributed flow-jamming attack is very low compared to that of the centralized efficient attack. This evaluation agrees with our intuition that the local linear programming approach leads to resource over-provisioning and a large increase in resource expenditure.

Fig. 10 illustrates the jamming impact and efficiency resulting from the heuristic flow-jamming algorithm in Fig. 5 using the same simulation parameters as that of Fig. 9. From Fig. 10(a), we see that the impact of the heuristic flowjamming attack is significantly reduced compared to that in Fig. 9(a) using the local linear programming approach. From Fig. 10(b), we see that the efficiency of the heuristic flowjamming attack is significantly increased compared to that in Fig. 9(b) using the local linear programming approach. This evaluation agrees with out intuition that a significant resource savings can be achieved by allowing a decrease in the jamming impact through the use of the conservative heuristic.

\section{CONCLUSION}

We presented and modeled the efficient flow-jamming attack in which an adversary selectively jams packets in network traffic flows. We proposed the evaluation metrics of jamming impact, efficiency, and resource variation and formulated optimal flow-jamming attacks with respect to these metrics using linear programming. We demonstrated the ability for a resource-constrained adversary to perform flow-jamming attacks efficiently with a significant impact on the network traffic flows. We showed that efficient flow-jamming attacks can be performed using distributed algorithms in the absence of centralized control of the jammers. Finally, we proposed two approaches for distributed flow-jamming depending on 


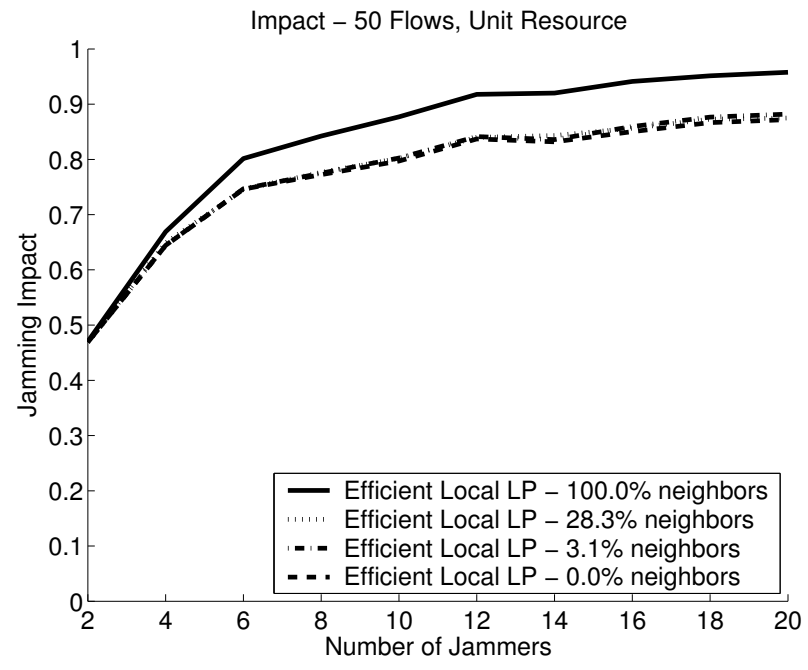

(a)

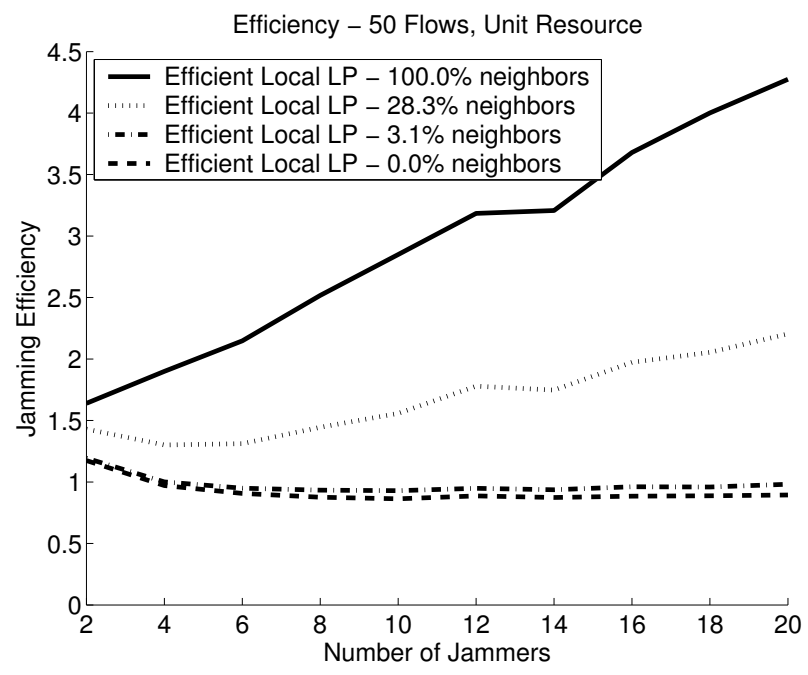

(b)

Fig. 9. We evaluate the effect of the distributed version of the efficient flowjamming attack using local linear programming as in Section IV-A in terms of the (a) jamming impact and (b) jamming efficiency as a function of the number of jammers $|\mathcal{J}|$ and the average neighborhood size as a percentage of $|\mathcal{J}|$.

whether the flow-jamming attack is aggressive or conservative with respect to jamming resource allocation.

\section{REFERENCES}

[1] R. Anderson, Security Engineering: A Guide to Building Dependable Distributed Systems. Wiley, 2001.

[2] R. A. Poisel, Modern Communication Jamming Principles and Techniques. Artech House, 2004.

[3] T. S. Rappaport, Wireless Communications: Principles and Practice, 2nd ed. Prentice Hall, 2001.

[4] J. Bellardo and S. Savage, "802.11 denial-of-service attacks: Real vulnerabilities and practical solutions," in Proc. USENIX Security Symposium, Washington, DC, Aug. 2003.

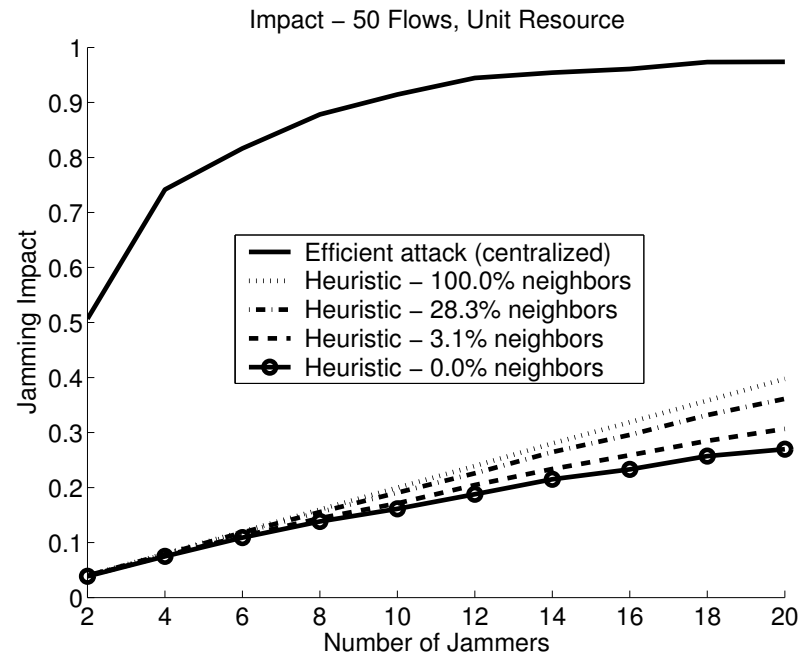

(a)

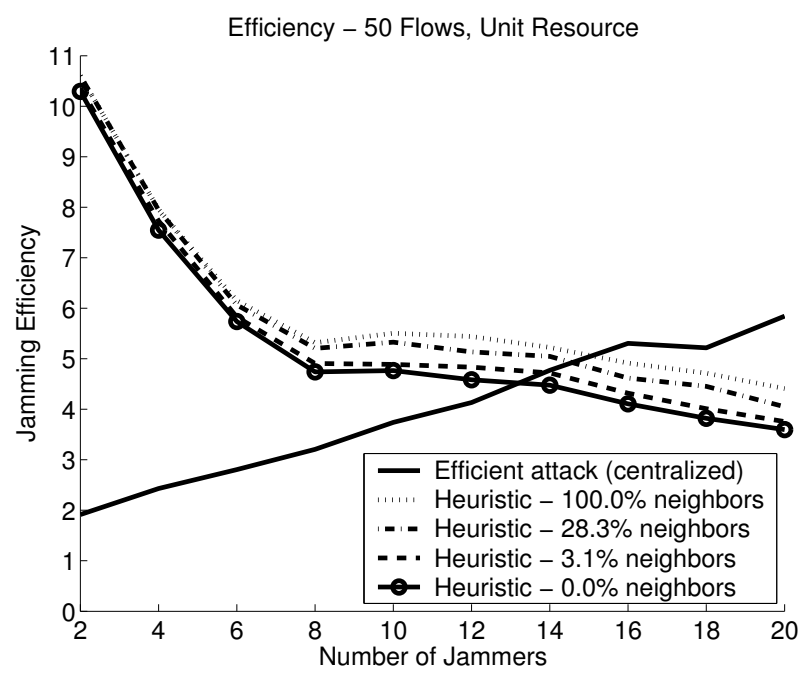

(b)

Fig. 10. We compare the centralized efficient flow-jamming attack in Section III-B to the heuristic attack in Section IV-B in terms of the (a) jamming impact and (b) jamming efficiency as a function of the number of jammers $|\mathcal{J}|$ and the average neighborhood size as a percentage of $|\mathcal{J}|$.

[5] D. J. Thuente and M. Acharya, "Intelligent jamming in wireless networks with applications to $802.11 \mathrm{~b}$ and other networks," in Proc. 25th IEEE Communications Society Military Communications Conference (MILCOM'06), Washington, DC, Oct. 2006.

[6] A. D. Wood and J. A. Stankovic, "Denial of service in sensor networks," IEEE Computer, vol. 35, no. 10, pp. 54-62, Oct. 2002.

[7] G. Lin and G. Noubir, "On link layer denial of service in data wireless LANs," Wireless Communications and Mobile Computing, vol. 5, no. 3, pp. 273-284, May 2005.

[8] R. K. Ahuja, T. L. Magnanti, and J. B. Orlin, Network Flows: Theory, Algorithms, and Applications. Prentice Hall, 1993.

[9] C. H. Papadimitriou and K. Steiglitz, Combinatorial Optimization: Algorithms and Complexity. Dover, 1998.

[10] R. A. Horn and C. R. Johnson, Matrix Analysis. Cambridge University Press, 1985.

[11] T. H. Cormen, C. E. Leiserson, and R. L. Rivest, Introduction to Algorithms. MIT Press, McGraw-Hill, 2000. 